\title{
The PMU-Based Power System Dynamic State Estimation Using Extended Kalman Filter
}

\author{
Xianing Jin ${ }^{1, a}$, Guanqun Wang ${ }^{2, b}$, Zhenyu Xue ${ }^{1}$, Chongbo Sun ${ }^{1}$, Yi Song $^{1}$ \\ ${ }^{1}$ State Power Economic Research Institute, State Grid, Beijing, 102209, China \\ ${ }^{2}$ Department of Electrical Engineering and Computer Science, Washington State University, \\ Pullman, 99163, USA \\ aemail: jinxianing@hotmail.com, bemail: guanqun.wang@outlook.com
}

Keywords: PMU; Dynamic State Estimation; Extended Kalman Filter

\begin{abstract}
Dynamic state estimation of power system is a sophisticated problem since voltage and current phasors under dynamic conditions are nonlinear and hard to be obtained. This paper presents a new power system dynamic state estimation method using Extended Kalman Filter (EKF) based on Phasor Measurement Unit (PMU). EKF can be used to deal with nonlinear system. With the help of PMU which is the key unit of Wide Area Measurement Systems (WAMS), continuous time waveforms with high accuracy and synchronized time stamps can be estimated. In case study, the effectiveness of the proposed method has been evaluated by dynamic state estimation of 3-bus powers system in Matlab, and scenarios with different PMU placement are compared. The proposed method achieves high accuracy in all these scenarios.
\end{abstract}

\section{Introduction}

In recent years, the number of Phasor Measurement Units (PMUs) installed on power grids has increased significantly. PMU can provide synchronized and high resolution data, thus is an enabling technology for power grids to perform wide area monitoring and control of system dynamics. The PMU data have already been introduced into power system state estimation [1-3].

Till now, most of the research focuses on static state estimation. However, besides static state variables, i.e., bus voltages and angles, it is also important to monitor power system dynamic states such as the rotor speed to ensure efficient and reliable operation and control of the grid. Since PMU data can be acquired to approximate the continuous time waveforms with high accuracy and synchronized time stamps, their installation can considerably improve monitoring of the system dynamics. The dynamics of the power system need to be modeled by a set of differential equations, and the PMU data can be treated as the measurements. The dynamic states of power systems can be reconstructed from the measurements using dynamic state estimation method, i.e., Kalman filter method.

In this paper, the dynamic model for power systems is established, and the PMU-based dynamic state estimation problem is formulated. Then the dynamic state estimation problem is solved by Extended Kalman filter method. A 3-bus power system has been studied as an example for the dynamic state estimation. The dynamic state estimation results for this system with different PMU placement are compared.

\section{PMU-Based Power System Dynamic State Estimation}

In this paper, the power system is reduced to internal generator nodes and the generators are modeled by classical second-order swing equations

$$
\begin{gathered}
\dot{\delta}_{i}=\omega_{i} \\
\dot{\omega}_{i}=\frac{1}{2 H_{i}}\left(P_{M_{i}}-P_{e_{i}}-D_{i} \omega_{i}\right), \quad i=1, \ldots, \mathrm{n}
\end{gathered}
$$


in which $\delta$ represents generator relative rotor angle, $\omega$ represents generator rotor angular speed. $P_{M}$ and $P_{e}$ are generator mechanical power and electrical power. $H$ is the generator inertia constant, and $D$ is the generator damping constant. All of the dynamic states and model parameters are in per unit. There are totally $n$ generators in the system.

A PMU measures bus voltage phasor and frequency at the bus, and current phasors on the incident lines. In this research, it is assumed that for generators where PMUs are installed, the rotor angle $\delta$ and rotor speed $\omega$ are measured by PMU, i.e.,

$$
z=\left[\begin{array}{ll}
\delta & \omega
\end{array}\right]^{T}
$$

In fact, the rotor speed $\omega$ can be obtained from bus frequency, and the rotor angle $\delta$ can be estimated in the substation level using the generator equivalent circuit

$$
\dot{E}^{\prime}=E^{\prime} \angle \delta=\dot{U}+\dot{I}\left(R+j X_{d}^{\prime}\right)
$$

in which $E^{\prime}$ represents the generator transient internal voltage phasor, $R$ and $X^{\prime}{ }_{d}$ are generator internal resistance and transient reactance. $\dot{U}$ and $\dot{I}$ are the external voltage and current phasor measured by PMU.

Then the power system dynamic model (1) with PMU measurements (2) can be represented in standard state space form

$$
\begin{aligned}
& \dot{x}(t)=f(x(t))+w \\
& z=h(x(t))+v
\end{aligned}
$$

where $x \in \mathrm{R}^{n}$ and $\mathrm{Z} \in \mathrm{R}^{p}$ represent state vector and measurement vector, $f$ and $h$ are the state function vector and measurement function vector, respectively. For (1) and (2), the state vector $x$ and output vector $z$ can be written as

$$
\begin{aligned}
& x=\left[\begin{array}{ll}
\delta^{T} & \omega^{T}
\end{array}\right]^{T} \\
& Z=\left[\begin{array}{ll}
\delta_{M}^{T} & \omega_{M}^{T}
\end{array}\right]^{T}
\end{aligned}
$$

\section{Dynamic State Estimation Using Extended Kalman Filter (EKF) Method}

The purpose for dynamic state estimation is to determine the dynamic state $x(t)$ based on the knowledge of the output $z$. One of the widely used method for dynamic state estimation is Kalman filter method. Since the model of system (4) is nonlinear, we cannot apply Kalman filter directly for dynamic state estimation. In this paper, the extended Kalman filter method [4] is applied to perform the dynamic state estimation. For system (4), if we use forward Euler method to solve the differential equations, the discrete form of (4) considering random errors is written by

$$
\begin{aligned}
& x_{k}=x_{k-1}+f\left(x_{k-1}\right) d t+w_{k-1}=\bar{f}\left(x_{k-1}\right)+w_{k-1} \\
& z_{k}=h\left(x_{k}\right)+v_{k}
\end{aligned}
$$

in which $d t$ is the time step for numerical integration and $\bar{f}\left(x_{k}\right)$ represents $x_{k}+f\left(x_{k}\right) d t$. $w_{k}$ and $v_{k}$ are the white Gaussian noise vector at time $k, w_{k} \sim N\left(0, Q_{k}\right), v_{k} \sim N\left(0, R_{k}\right)$. The EKF for this nonlinear system is 


$$
\begin{aligned}
& \hat{x}_{k \mid k-1}=f\left(\hat{x}_{k-1 \mid k-1}\right) \\
& P_{k \mid k-1}=F_{k-1} P_{k-1 \mid k-1} F_{k-1}^{T}+Q_{k-1} \\
& y_{k}=z_{k}-h\left(\hat{x}_{k \mid k-1}\right) \\
& S_{k}=H_{k} P_{k \mid k-1} H_{k}^{T}+R_{k} \\
& K_{k}=P_{k \mid k-1} H_{k}^{T} S_{k}^{-1} \\
& \hat{x}_{k \mid k}=\hat{x}_{k \mid k-1}+K_{k} y_{k} \\
& P_{k \mid k}=\left(I-K_{k} H_{k}\right) P_{k \mid k-1}
\end{aligned}
$$

in which

$$
\begin{aligned}
& F_{k-1}=\left.\frac{\partial \bar{f}}{\partial x}\right|_{\hat{x}_{k-1 k-1}} \\
& H_{k}=\left.\frac{\partial h}{\partial x}\right|_{\hat{x}_{k \mid k-1}}
\end{aligned}
$$

are the Jacobians of the nonlinear equations. The initial value for EKF is

$$
\begin{aligned}
& \hat{x}_{0 \mid 0}=E\left[x\left(t_{0}\right)\right] \\
& P_{0 \mid 0}=\operatorname{var}\left[x\left(t_{0}\right)\right]
\end{aligned}
$$

With this recursive process, the dynamic state $x(t)$ can be estimated at each step as $\hat{x}_{k \mid k}$.

\section{CASE STUDY}

\section{(1) Bus System}

In this paper, a 3-bus system with lossless transmission lines is used to illustrate the dynamic state estimation problem, which is shown as Fig. 1.

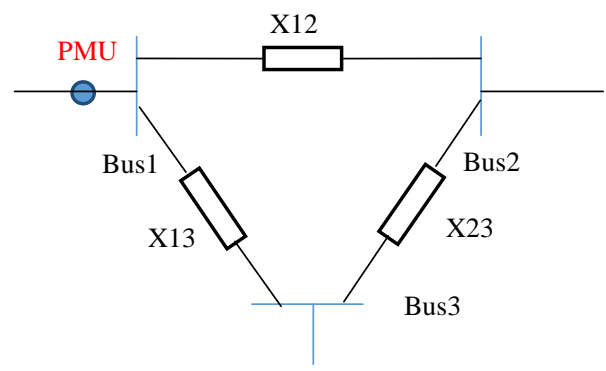

Figure 1. A simplified 3-bus sytem

The dynamic model of this system, which is shown as follows, is formulated by classical rotor dynamic equations, where generator rotor angle and rotor speed are the state variables. 


$$
\begin{aligned}
& \dot{\delta}_{1}=\omega_{1} \\
& M_{1} \dot{\omega}_{1}=P_{M 1}-\frac{E_{1} E_{2}}{X_{12}} \sin \left(\delta_{1}-\delta_{2}\right)-\frac{E_{1} E_{3}}{X_{13}} \sin \left(\delta_{1}-\delta_{3}\right)-D_{1} \omega_{1} \\
& \dot{\delta}_{2}=\omega_{2} \\
& M_{2} \dot{\omega}_{2}=P_{M 2}-\frac{E_{1} E_{2}}{X_{12}} \sin \left(\delta_{2}-\delta_{1}\right)-\frac{E_{2} E_{3}}{X_{23}} \sin \left(\delta_{2}-\delta_{3}\right)-D_{2} \omega_{2} \\
& \dot{\delta}_{3}=\omega_{3} \\
& M_{3} \dot{\omega}_{3}=P_{M 3}-\frac{E_{1} E_{3}}{X_{13}} \sin \left(\delta_{3}-\delta_{1}\right)-\frac{E_{2} E_{3}}{X_{23}} \sin \left(\delta_{3}-\delta_{2}\right)-D_{3} \omega_{3}
\end{aligned}
$$

The parameters of the 3-generator system are given in [5]. Assume there is a three-phase fault followed by normal clearing on transmission line 1-3. During the fault, the dynamical system in (10) is changed since branch $1-3$ is disconnected. Then it is assumed that the breaker recloses successfully at $1 \mathrm{~s}$ and the system topology is recovered.

\section{(2) Dynamic State Estimation}

Assume that there is one PMU at bus 1 and the corresponding states of $\delta_{1}$ and $\omega_{1}$ are directly monitored. The EKF is set as follows.

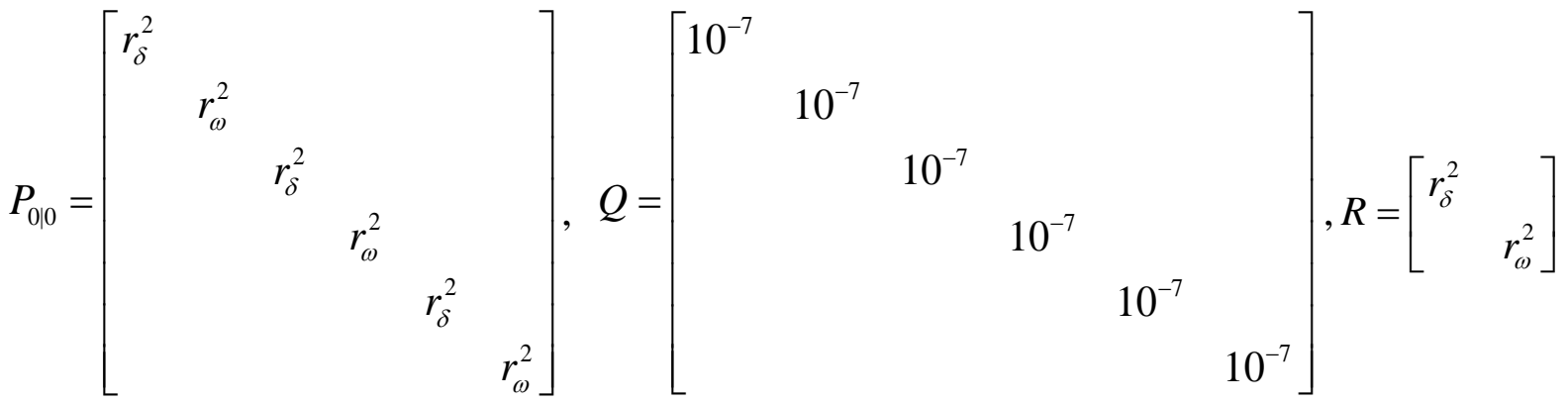

$r_{\delta}$ and $r_{\omega}$ denotes the error of $\delta$ and $\omega$, set as $0.5 \pi / 180$ and $10-3 \omega_{0}$. The time step $d t$ is $0.01 \mathrm{~s}$. The dynamic estimation is performed after the breaker is reclosed. The black dash lines denote real states, red solid lines denote estimated states, and blue dots denotes the measurements from PMUs.
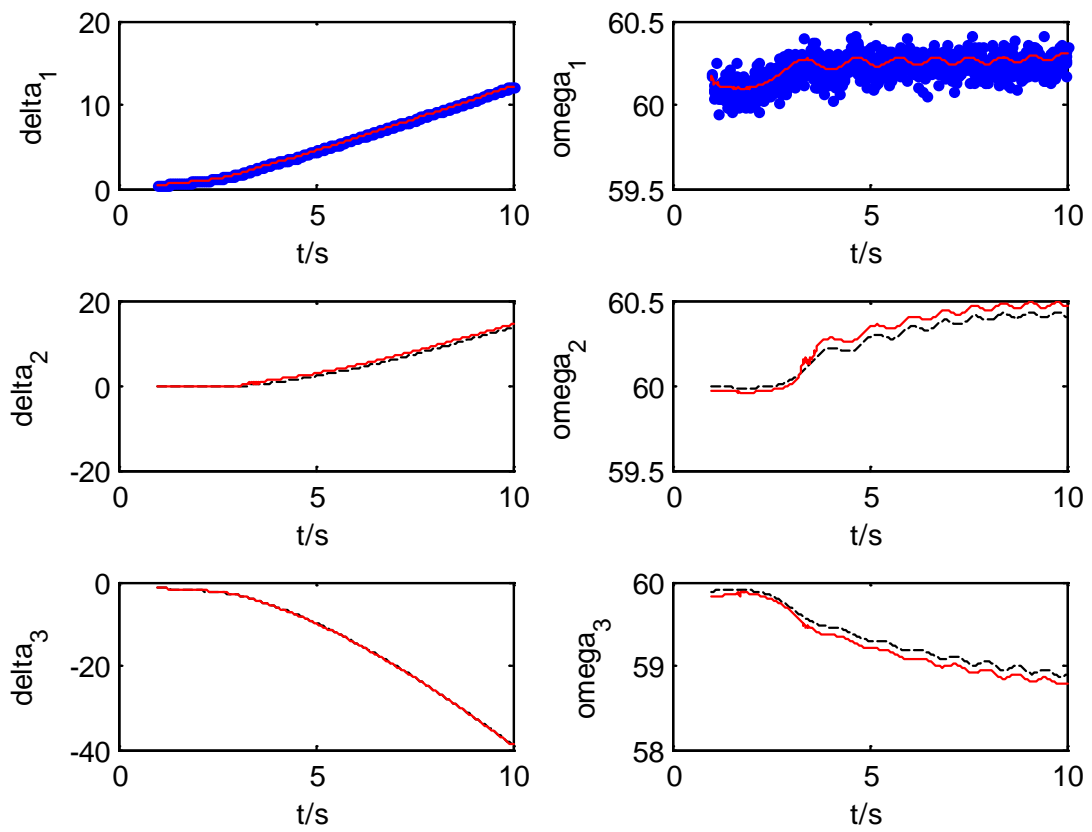

Figure 2. Estimation results for placing PMU at generator 1 for 3-bus system

From Fig. 2, it shows that although the PMU measurements have some errors, the EKF can 
estimate the dynamic states with promising accuracy.

When PMUs are placed at different buses, the results are shown as Fig. 3 and 4.
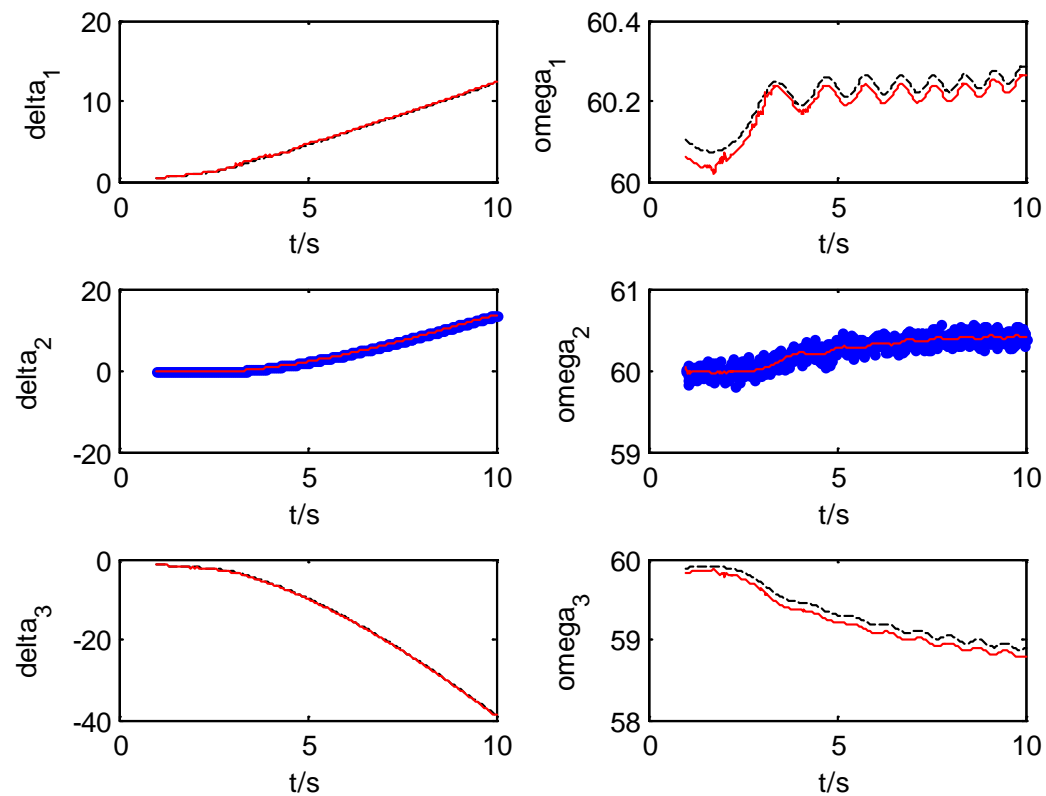

Figure 3. Estimation results for placing PMU at generator 2 for 3-bus system
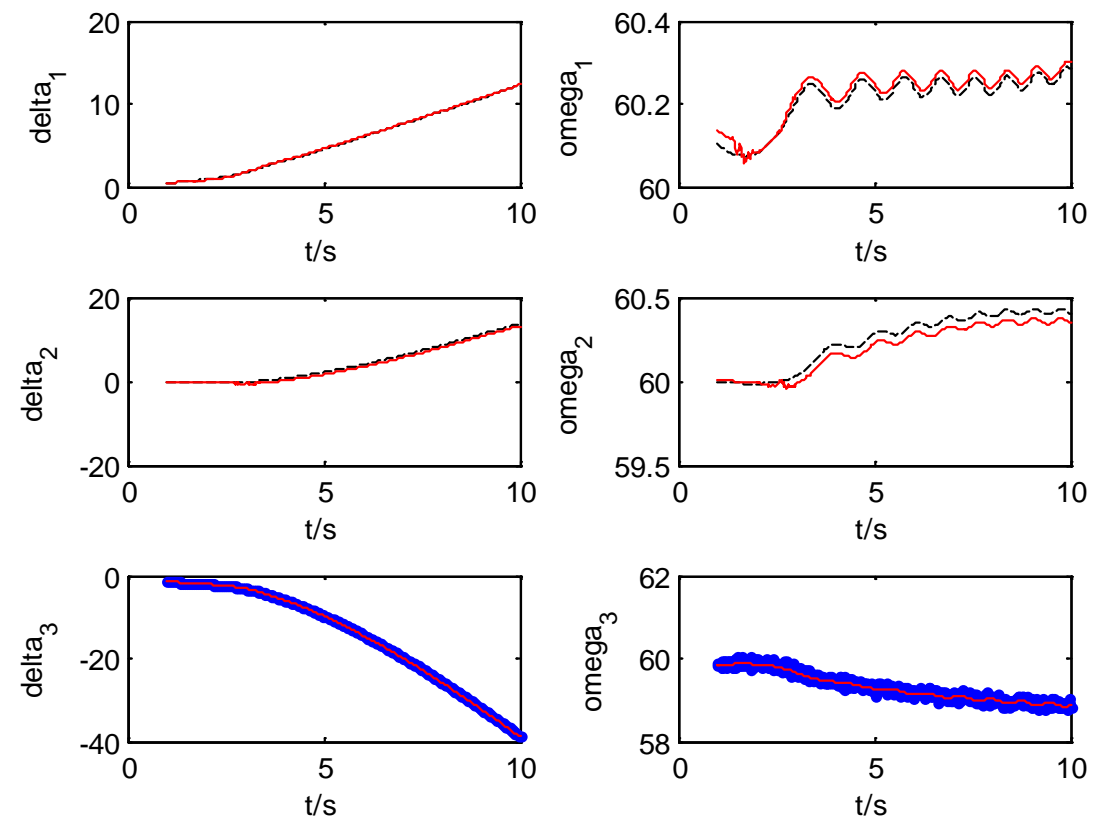

Figure 4. Estimation results for placing PMU at generator 3 for 3-bus system

\section{Conclusion}

In this paper, the extend Kalman filter method is applied for power system dynamic state estimation based on PMU. A 2-order dynamic model is formulated to represent the dynamic system. The EKF are used to solve this nonlinear problem, and PMU is good at synchronized phasor measurement with time stamps. A 3-bus power system is simulated to evaluate the effectiveness of the proposed method for dynamic state estimation. It demonstrated that the proposed method can be an effective tool for applications such as wide-area stability analysis, modal analysis and power system emergency control. 


\section{References}

[1] A. G. Phadke, J. S. Thorp, and K. J. Karimi, "State Estimation with Phasor Measurements," IEEE Transactions on Power Systems, vol. 1, no. 1, pp. 233-238, 1986.

[2] S. Chakrabartiand and E. Kyriakides, "Optimal Placement of Phasor Measurement Units for Power System Observability”, IEEE Transactions on Power Systems, vol. 23, no. 3, pp. 1433-1440, 2008.

[3] R. F. Nuqui and A. G. Phadke, "Phasor Measurement Unit Placement Techniques for Complete and Incomplete Observability," IEEE Transactions on Power Delivery, vol. 20, no. 4, pp. 2381-2388, 2005.

[4] E. Ghahremani, I. Kamwa, "Dynamic State Estimation in Power System by Applying the Extended Kalman Filter With Unknown Inputs to Phasor Measurements," IEEE Transactions on Power Systems, vol. 26, no. 4, pp. 2556-2566, 2011.

[5] J. Yan, C. -C. Liu, U. Vaidya. "PMU based monitoring of rotor angle dynamics." IEEE Transactions on Power Systems, vol. 26, no. 4, pp. 2125-2133, 2011. 